Horizontes. Revista de Investigación en Ciencias de la Educación ISSN 2616-7964

abril-junio, 2017

\title{
Gerencia de la educación adaptada a la realidad venezolana para el año 2017
}

\author{
Management of education adapted to the Venezuelan reality for the \\ year 2017 \\ Leonardo Fabio Vásquez Gómez \\ leonardofabio0707@gmail.com \\ Universidad Militar Bolivariana de Venezuela
}

Recibido: 20-10-2016 / Revisado: 25-10-2016 / Aceptado: 13-11-2016 / Publicado: 10-04-2017

\begin{abstract}
RESUMEN
El presente estudio propone los aspectos a considerar en la gerencia de la educación venezolana adaptada a la realidad que se vive en el año 2017. Se inicia con un resumen diacrónico sobre la evolución de la educación considerando las culturas más destacadas a lo largo de la historia, que nos ilustran y que obedece a la organización actual del preescolar (o kindergarten), la escuela, el liceo y las Universidades. Posteriormente, teniendo como base la estructura educativa de Alemania, China, Japón, España y Canadá, países que, según el Programa Internacional para la Evaluación de Estudiantes o Informe PISA (por sus siglas en inglés: Programme for International Student Assessment), el rendimiento académico de los alumnos en matemáticas, ciencia y lectura, son los más destacados en el nivel educativo mundial. Partiendo de la premisa del informe PISA, de conformidad con los aspectos más destacados que han garantizado el éxito en la educación de estos países y en contraste con la realidad actual de Venezuela se plantean los diez aspectos que limitan la gerencia adecuada de la educación venezolana y finalmente se proponen las recomendaciones para mejorar nuestra educación.
\end{abstract}

Palabras clave: Educación; evolución; rendimiento académico; nivel; realidad; adaptación
ABSTRACT

The present study proposes the aspects to consider in the management of Venezuelan education adapted to the reality that is lived in the year 2017. It begins with a diachronic summary on the evolution of education considering the most outstanding cultures throughout history, that illustrate us and that obeys the current organization of the preschool (or kindergarten), the school, the lyceum and the Universities. Subsequently, based on the educational structure of Germany, China, Japan, Spain and Canada, countries that, according to the International Program for Student Evaluation or PISA Report (for its acronym in English: Program for International Student Assessment), performance The academic content of students in mathematics, science and reading are the most outstanding in the world education level. Starting from the premise of the PISA report, in accordance with the most outstanding aspects that have guaranteed the success in education of these countries and in contrast with the current reality of Venezuela, the ten aspects that limit the adequate management of Venezuelan education and Finally recommendations are proposed to improve our education.

Key words: Education; evolution; academic performance; level; reality; adaptation 


\section{INTRODUCCIÓN}

La gerencia de la educación adaptada a la realidad, es una percepción personal como docente y como alumno del autor. Con la finalidad de motivar y aprender las bondades de la educación se presenta un recuento histórico de la importancia que a través de la historia se ha evidenciado que la educación es sin duda parte la evolución de cada nación o civilización.

Se inicia la cronología con la civilización griega por ser esta, referencia obligatoria en el aspecto filosófico y doctrinario además de seguir vigentes sus postulados. En la actualidad nos muestran que cuando una civilización orienta todo a la educación de sus ciudadanos, sus legados trascienden $\mathrm{y}$ perduran a través del tiempo. También, se da una pequeña terminología básica sobre conceptos que en materia de educación hay que tener siempre presentes y muy bien definidos.

Fundamentándose en las fuentes abiertas del internet, se explican cómo funcionan los sistemas educativos en los países donde según el Informe del Programa Internacional para la Evaluación de Estudiantes o Informe PISA (por sus siglas en inglés: Programme for International Student Assessment), que mide el rendimiento académico de los alumnos en matemáticas, ciencia y lectura. Entre ellos están: Alemania, Japón, China, España y muy resumidamente Canadá.

Posteriormente, se muestra la perspectiva a título personal del autor sobre la realidad de la educación en Venezuela para el año 2017 y lo enmarca en 10 puntos que, a pesar de no ser una investigación de campo con encuestas, no escapan de la realidad, de hecho, tampoco se basa en estadísticas nacionales e internacionales por considerarlas el autor, datos susceptibles y manipulables por los intereses de cada agencia.
Finalmente, el autor describe en ocho puntos su propuesta para la gerencia de la educación de la actualidad.

\section{La motivación de aprender y educarse}

"La educación es la trasmisión de la civilización”. Will Durant (1885-1981) Con el fin de hacer dinámica la lectura del presente libro y dar una justificación al tema la Gerencia de la Educación adaptada a la realidad, se pretende ir más allá de simples conocimientos teóricos que se encuentran en los textos y en el internet; dando, por ende, una perspectiva histórica de lo que ha sido la evolución de la educación.

Los acontecimientos y hechos que han influido en la historia de la humanidad, desde la aparición de la escritura hasta nuestros días, nos demuestran la necesidad de estudiar el pasado, para comprender el presente y mejorar el futuro. La Historia, entendida como ciencia, nos invita a reflexionar sobre nuestro origen, evaluar nuestra realidad y apreciar hacia dónde vamos.

Se considera que la educación se encuentra ligada al avance y progreso de cada civilización, de allí, que es de obligatoriedad iniciar esta reseña por la civilización griega; al respecto, Arteola (2010) hace referencia al hecho de que el inicio educativo se generó con un cambio en las bases educativas y de pensamiento, dando paso a los centros educativos como el Liceo y la Academia. Filósofos como Sócrates, quien enseñó a través de la Mayéutica; Platón, fundador del liceo; Heráclito, padre de la dialéctica, y Aristóteles fue quien desarrolló los valores y virtudes cívicas para todos, y no exclusivamente para las clases privilegiadas.

Ellos influenciaron con sus postulados trascendiendo y transmitiendo a todo el mundo occidental del momento, los preceptos educativos. Para la civilización griega en sus inicios la finalidad de la educación partía del ciudadano que necesitaba la República; por 
tanto, en Esparta se formaban soldados o guerreros con fortalezas físicas y estoicismo, que fueran capaces de defender con honor y patriotismo su ciudad conocida y llamada la Polis. En Atenas, por el contrario, se desarrolló una educación más centrada hacia lo académico, la misma buscaba formar hombres intelectuales bajo la premisa de un desarrollo intelectual del pensamiento y en el espíritu.

Es importante resaltar, en ese apartado que, así como para Platón el fin último de la educación era la felicidad, y por ello hablaba del dualismo: cuerpo y alma. Para Aristóteles, lo era el desarrollo de los valores y las virtudes cívicas, entendiendo entonces que la educación debía ser impartida a toda la población y no sólo a un grupo social privilegiado.

Ahora bien, continuando con este recorrido histórico, encontramos que la edad media se caracterizó por la apertura de varias universidades en países como Italia, España, entre otros; generándose intercambios académicos entre estudiantes que viajaban libremente de una institución a otra. Al respecto, es importante señalar a Fernández \& Clara (2010) cuando señalan que tanto las universidades del norte de Europa, como las de París, Oxford, y Cambridge, eran administradas por los profesores; mientras que las del sur, como la de Bolonia (Italia) o Palencia y Alcalá en España, lo eran por los estudiantes.

La educación medieval, desarrolló una forma de aprendizaje a través del trabajo o servicio propio; resaltando entonces, que durante toda la edad media, existieron aportes de judíos y musulmanes. Se destaca entonces, como los centros de Toledo y Córdoba en España atrajeron a estudiantes de todo el mundo civilizado durante la época.

En el Renacimiento, muchos profesores del área de lengua y literatura griega emigraron desde Constantinopla a Italia, a desarrollar estos conocimientos. Entre algunas de las personalidades del renacimiento que contribuyeron al desarrollo de la praxis educativa de la época, sobresalen el humanista alemán Erasmo de Rotterdam, el educador alemán Johannes Sturm, el ensayista francés Michel de Montaigne y el humanista y filósofo español Luis Vives. Cuyos aportes, dan paso a las primeras universidades americanas fundadas en Santo Domingo (1538), en México y en Lima (1551). El desarrollo sostenido de cada uno de estos modelos educativos se constituyó en el sistema de enseñanza secundaria que perduraría hasta el siglo XX.

En cuanto a los aportes de la Iglesia, como institución desarrolladora de procesos educativos, se destacan los desarrollados por los Jesuitas y los Salesianos. Por su parte, los Jesuitas, como se conoce a los miembros de la congregación de la Compañía de Jesús, fundada a mediados del siglo XVI (1534) por el español Ignacio de Loyola (1491-1556) como una organización eclesiástica católica, con principios de régimen militar para combatir en el plano ideológico a los reformadores protestantes y al movimiento humanista; promovieron un sistema de escuelas que ha tenido un papel preponderante en el desarrollo de la educación católica y moral en muchos países desde el siglo XVI. Por otro lado, ha existido un importante aporte por parte de los Hermanos Salesianos de la obra de Don Bosco, principalmente en la Patagonia, en cuanto a la educación católica y de servicio al prójimo.

Continuando con el recorrido histórico, se hace necesario destacar en el siglo XVII, al filósofo francés René Descartes, quien subrayó el papel de la lógica como el principio fundamental del pensamiento racional, postulado que se ha mantenido hasta la actualidad como base de la educación en Francia. En el siglo XVIII, llamado el Siglo de las Luces, el teórico más relevante fue JeanJacques Rousseau, cuya influencia en la praxis educativa fue considerable tanto en Europa 
como en otros continentes. De acuerdo a, Fernández \& Clara (2010), una crítica que le hacen algunos sectores a Rousseau, fue la falta de aplicabilidad de algunas ideas, cayendo muchas veces en un enfoque muy teórico. Es importante, mencionar en este punto, que el Siglo de las Luces, vio el surgimiento de las logias masónicas como agentes de cambio en la ruptura social, poniendo como centro de la vida, al hombre libre y autodeterminado por la razón; alimentado evidentemente por su ilustración, vale decir por su educación.

Posterior a la época medieval, la Iglesia Católica, Apostólica y Romana cobra fuerza en la educación. Los líderes de la Iglesia comienzan a fundar seminarios y monasterios, impartiendo una educación enfocada al trabajo y al servicio hacia los demás. Esta educación era exclusiva para sus alumnos, porque solo podían optar a ser sacerdotes o clérigos. Posteriormente, a solicitud de la realeza, también se instruyó a los nobles permitiendo con los años la fundación de las universidades.

Es importante destacar, que el ciudadano Medieval debía ser culto, retomando lo clásico de la época greco-romana pero creyente en la fe católica. En esta época el desarrollo de las ciencias llevó a lo que se ha conocido como el "Descubrimiento de América", generado por los avances en el área de la astronomía, la cartografía y la navegación. La educación, continuaba siendo tradicionalista, memorística y centrada en el maestro y no en el alumno; enfocada en la formación del hombre y no de la mujer.

Dos siglos después, el aporte más importante de la educación contemporánea lo constituye la creación del Kindergarten (Jardín Infantil) fundado por Friedrich Froebel, educador alemán, que tomando algunas ideas de Pestalozzi (con quien trabajó por unos años en Suiza) y basándose en su experiencia como profesor, escribió el libro "La Educación del Hombre" en 1806. En este libro, se reconoce la importancia de la actividad del niño en su propio aprendizaje y la idea del juego como un instrumento para desarrollar las habilidades y conocimientos que le permiten relacionarse con el mundo. De allí, que todo el arte de la educación se encuentre basado en el conocimiento profundo y en la aplicación de esta ley, única capaz de contribuir al desarrollo y expansión del ser inteligente, y única susceptible de conducir a éste a la consumación de su verdadero destino.

Hacia el año de 1837, Froebel fundó el primer "Instituto para el Juego y el Cuidado de Niños Pequeños" en BadBlankeburg (Alemania), al que posteriormente denominó Kindergarten; su lema era "respetar la actividad creadora del niño en un clima de libertad y en contacto con la naturaleza". En este lugar los niños cantaban, bailaban y jardineaban. Se preocupó de la educación infantil preescolar, ya que en esta etapa de desarrollo del niño(a), se desarrollan destrezas que le servirán en los años siguientes de estudio y formación.

Por su parte, María Montessori (18701952) educadora y pedagoga italiana, decía que "los niños son como esponjas"; de allí que creara un método que no sólo es aplicable a los niños(as) de la etapa de preprimaria, sino también a estudiantes de primaria, secundaria, universidad y también para niños con capacidades diferentes.

Un aspecto importante del desarrollo educativo de la etapa contemporánea han sido los precursores de la Escuela Nueva o Activa, en donde ya la educación no es centrada en el maestro sino en el alumno. Otro gran aporte lo constituyó la teoría constructivista de Piaget (1896-1980) y su definición de las diferentes etapas de desarrollo del niño(a) preescolar, el de nivel primario y adolescentes. También Alfred Adler (1870-1937) hablaba de la benevolencia amistosa con los alumnos(as), respetando su individualidad y su integridad; 
además de proponer el servicio a los demás y procurar el bien común.

En la actualidad, pensadores modernos como Paulo Freire y Edgar Morín proponen enfoques de la educación con respecto al aprendizaje permanente e integral de la persona. Por otro lado, el surgimiento de la educación no formal y el concepto de andragogía, como modelos de estudio para jóvenes y adultos con el fin de continuar su prosecución escolar, se mantiene como un enfoque de formación y educación permanente; ya sea en centros de formación profesional $o$ en instituciones más sistematizadas como las universidades.

La educación es en sí misma un proceso dinámico, por lo que presenta cambios permanentes $y$ continuos en toda su estructura. De esta manera, se puede afirmar que la educación desde cualquier modalidad, procura generar un aprendizaje a través de la interacción del ser humano con el medio ambiente, convirtiéndose entonces, en el resultado de la experiencia. El medio ambiente estará determinado por un momento histórico determinado, que rige una sociedad y una estructura política acorde con el mismo. De allí que sea la historia la encargada de describir ese momento de la vida del ser humano, entendiendo el porqué de los hechos; es decir, las causas y las consecuencias de los mismos.

Ahora bien, una vez desarrollada esta síntesis de lo que ha sido la praxis educativa en la historia de la humanidad, se presenta una terminología que le facilita al lector adentrarse en la apropiación de términos relacionados con el conocimiento de la educación y la gerencia educativa.

\section{Realidad de la Educación Venezolana para el año 2017}

Luego de hacer el repaso cronológico muy superficial de la historia de la educación y posterior al análisis de los principales sistemas educativos del mundo; es el momento de apartarnos de las referencias bibliográficas y de las páginas de internet para exponer la perspectiva actual de la educación venezolana para el año 2017.

Este no es un trabajo de campo, por lo que no tiene encuestas. Tampoco se quiere evocar el trabajo de los investigadores y docentes de los últimos veinte (20) años en las universidades venezolanas, quienes teóricamente han abordado este tema de la educación. La propuesta de este libro es que no podemos afirmar si la educación pasada fue mejor o peor... podemos afirmar que la educación funcionaba mejor.

Esta afirmación obedece que al leer y revisar la ley de educación venezolana actual, los planes de estudio de las universidades, los contenidos sinópticos y analíticos de las unidades curriculares presentan un compendio holístico, filosófico y pedagógico muy integral y completo, sin embargo $y$ tristemente se puede apreciar en más de un cincuenta $(50 \%)$ por ciento (en la mejor hipótesis de optimismo), un alumno regular desconoce el contenido de lo que se le instruyó y menos aún demuestra o domina los objetivos planeados y planteados en cada programa, materia o unidad curricular.

Todos los países (Alemania, China, Japón, España y Canadá) con alto rendimiento académico coinciden que la Educación Inicial, preescolar, básica o incluso los Kindergarten están orientados a la Formación Social del Ciudadano. El conferencista colombiano Yokoi Kenji confiesa que su padre (de origen japonés) le decía: "la disciplina tarde o temprano vence a la inteligencia".

El conferencista Yokoi Kenji, en sus videos de YouTube, nos pone grandes ejemplos de cómo la educación japonesa se basa en la disciplina más que en la inteligencia. Aun cuando mostramos a Japón, Alemania, China, España y Canadá como los países con la mejor educación no es porque son más inteligentes, por supuesto que no, es que ellos le han dado continuidad a su proceso 
educativo. Le han dado la importancia que merece la educación en los primeros años del estudiante y le dan un acompañamiento para brindarle la asesoría que le permita orientar eficientemente al estudiante en lo que va hacer su futuro profesional.

Amigo lector reflexione lo siguiente: Estudiando en Venezuela ¿Cuántos estudiantes disciplinados conoce usted? ¿Cuántos estudiantes que haya conocido se dedican íntegramente a estudiar diariamente? ¿Cuántos jóvenes entre 10 y 20 años usted considera respetuosos, educados y virtuosos? No dudamos que existan, pero son casos excepcionales 1 ó 5 de cada 100. Siendo esto así ¿Cómo le exigimos a la sociedad si no formamos ciudadanos?

A continuación, se van a enumerar diez aspectos reales de la actualidad de nuestra educación que, aun teniendo el fundamento epistemológico, teórico y doctrinal en sus documentos rectores, impiden aumentar la calidad de la educación:

1. Descomposición social: Los estudiantes provienen de hogares generalmente fracturados o disfuncionales que, aunado a la difícil situación económica de un alto porcentaje de la población, exige que todos los miembros de la familia trabajen, lo que incide en el rendimiento académico y en la poca o mala orientación de los padres o representantes a reforzar el proceso educativo del niño. Ante esta situación, que como citamos al principio en vez de avocarnos a formar un CIUDADANO EJEMPLAR, el joven estudiante carece de PATRONES y ejemplos de ciudadanos. Ni siquiera se presentan muestras estadísticas por no considerarlas confiables, lo cierto es que el entorno social en Venezuela nos muestra que los valores religiosos, sociales y culturales están en detrimento y que EDUCARSE pasó a ser un segundo plano; es más valioso tener dinero, vehículos, bienes $\mathrm{y}$ un buen cargo en una institución o empresa que dedicarse a estudiar algo. Se dice en términos coloquiales ¿para qué estudiar?... eso no da plata.

2. Calidad docente: Ser docente en otros países requiere un amplio estudio $\mathrm{y}$ dedicación por parte del educador. En Venezuela usted con tener la mínima nota aprobatoria en la carrera de educación, se gradúa y está acreditado para dar clases a nivel nacional y ejercer la labor docente. En otros países el docente cuenta con una remuneración que está entre los cinco mejores empleos remunerados de ese país, lo que les permite a los docentes una calidad de vida digna y que les permite dedicarse a su profesión exclusivamente, incluso a profundizar y mejorar los procesos educativos. Aunado al primer punto enumerado, ser docente representa en los países élites de la educación, una forma de vivir confortablemente, el estudiante ve en su docente un modelo de vida. En la Venezuela entre 1870 y 1990 un docente contaba con una seguridad social que le permitía vivir también con un nivel de vida confortable. En la actualidad, los docentes están sobreviviendo o subsistiendo con recursos y trabajos extras que le permitan satisfacer sus necesidades y el aula y la instrucción son un obstáculo para su subsistencia.

3. La influencia del docente: El docente de los países con alto rendimiento educativo, asesora $\mathrm{y}$ orienta a los padres $\mathrm{y}$ al estudiante sobre el futuro del estudiante. Es decir, el docente INFLUYE notablemente en la vida del estudiante. A través de sus instrumentos y de su pedagogía, el docente o profesor logra discernir e identificar las actitudes y cualidades de sus estudiantes más aptas para su futuro. A esta influencia los padres acceden y apoyan el trabajo docente. En Venezuela los alumnos estudian lo que quieren con o $\sin$ condiciones, solo van a clases y cumplen 
con las asignaciones que aun cuando no tenga las capacidades y condiciones no hay nada que el internet o algún conocedor de la materia no lo facilite como un favor o a cambio de cierto monto de dinero. Hay testimonios por las redes sociales, incluso casos que por confidencialidad no se exponen las fuentes que confirman que en las escuelas primarias y liceos ubicados en sectores populares, los docentes son víctimas de robos, atracos y amenazas de muerte por parte de sus alumnos o de los familiares de ellos, que ante los casos de aplazar al alumno se ven obligados a promoverlos de año, calificarlos satisfactoriamente y eximirlos de actuar disciplinaria o legalmente ante las faltas. Incluso hay delitos cometidos por estos alumnos.

4. Control de asistencia a clases: Para los países con alto nivel educativo, la legislación en materia educativa para el niño es estricta. Por ejemplo, en Inglaterra, cuando el docente nota la inasistencia de un estudiante, procede a llamar de inmediato a su representante para que justifique la ausencia del estudiante; sino es justificada, un tribunal procede a tomar acciones legales en contra de ese representante. Le dejamos al lector le recomendación de entrevistar a cualquier docente de cualquier nivel educativo en Venezuela, si el alumno asiste, está bien, si no asiste, también está bien, porque el docente (en su mayoría) se alegra y se siente mejor con menos estudiantes, no tiene un contacto directo con los padres o representantes y si hay que justificar siempre es fácil coordinar un reposo médico (verdadero o inventado). Incluso podemos hacer referencia que hay docentes que califican la asistencia. Ciertamente la Ley Orgánica de Protección del Niño y el Adolescente de Venezuela, garantiza la seguridad escolar a los niños y adolescentes estudiantes, pero ¿dónde están los órganos ejecutores que controlen este aspecto vital, la asistencia? Hay niños que tienen la necesidad de ir a trabajar y no existe organismo que controle esto, de hecho, en fechas y horarios escolares en Venezuela, es normal ver niños ejerciendo actividades laborales y comerciales y nadie dice nada.

5. La filantropía: Este punto data desde el Manifiesto de Cartagena del Libertador Simón Bolívar en 1812, en este documento, Bolívar, expone que una de las causas de la caída de la primera república era "la Filantropía por legislación...” y es común en Venezuela oír la siguientes frases: "pobrecito el niño (a)...", "él estudia pero no entiende...", "va a tocar pasarlo así...", "profesor(a) haga una excepción con él", "dele esta oportunidad que él después aprende", y la mejor de todas, "para qué le exige eso si no le va a servir para el futuro". Y así como estas frases muchas otras que hacen que nuestra educación en términos generales se vea afectada negativamente. Académicamente, se puede apreciar mala base matemática, gramática, histórica y ante tantas deficiencias surge la pregunta ¿Cómo llegó el alumno "X" al nivel "Y"? y más complejo aún ¿cómo logran obtener un título académico si como ciudadanos carecen de tantas aptitudes? La Educación está orientada a formar al ciudadano, hacerlo un hombre útil, que entienda que forma parte de una sociedad a la que debe tributar en beneficio de todos y no de un egocentrismo particular.

6. Mecanismos de evaluación: Continúa el ejemplo entre los países de elevado nivel académico y Venezuela. En los países modelos se diseña una prueba a nivel nacional o regional que permite determinar el grado cuantitativo de sus estudiantes, basado en la formulación de preguntas objetivas que estadísticamente arrojan resultados que permite clasificar a sus estudiantes en sus distintas áreas de 
evaluación y de conocimiento. En Venezuela, solo existe la Prueba de Aptitud Académica que funge para determinar parte de los cupos para el acceso o ingreso a las universidades. En el resto de los centros de educación todo funciona de acuerdo al ritmo que le imprima su decano, rector o director. No hay una continuidad en el proceso, no tenemos un control o una herramienta efectiva que nos determine nuestro índice académico (real), el nivel de coeficiente intelectual de nuestros estudiantes. Contamos con excelentes planes, leyes y programas de educación, lo que carecemos es de controles y estadísticas confiables, carecemos de mecanismos de control que nos indiquen las fallas, mejoras o áreas de especial atención.

7. La distorsión cultural: Somos venezolanos, somos mestizos, tenemos una música, un folklore, una historia pero que lamentable es ver nuestra juventud tratando de ser y hacer lo que hacen otras naciones: Halloween, muñecos de nieve, música (si se le puede llamar así) interpretada por personajes cuya trayectoria carece de una formación musical o educativa y no son más que estereotipos, con la ropa y apariencia que adineradas organizaciones promueven como LA MODA. En la entrega del premio Oscar del año 2015 se determinó que de los más de mil actores en el recinto donde se hizo la premiación no se computaban más de ochenta años en preparación académica de todos los actores. Lo asombroso de esto es que más del $50 \%$ de la fortuna mundial se encontraba en las cuentas bancarias y bienes de estos personajes. Así pues, vemos como a nivel mundial el estudio no es la base que garantice la vida que todos quieren tener, los lujos que todos quieren disfrutar $\mathrm{y}$ nuevamente cobra vida la frase ¿para qué estudio si eso no me a dará dinero?

8. Lectura, escritura y matemática: Si te recomiendan un libro, inmediatamente la pregunta es ¿lo puedo descargar de internet? Es más barato, es más práctico, pero los estudiantes venezolanos en un muy bajo porcentaje leen. Encueste a sus familiares, amigos de trabajo, estudiantes de cualquier nivel y pregunte cuantas obras han leído: Doña Bárbara, lanzas coloradas, obras de nuestros precursores de la independencia, ¿poesía? ¿ópera? No, para muchos es aburrido, mejor vamos al cine o enviamos un mensaje o video a través de las redes sociales y de esta manera somos autómatas de un sistema que sustituyó las palabras por emojis. ¿Calcular una cuenta? Mejor saquemos la calculadora y es más fácil y rápido y el ábaco pasó a ser un objeto digno de museo. ¿Calcular un espacio, área o capacidad de alguna figura geométrica? Es mejor descargar la aplicación de internet. En conclusión, la vida es fácil, busquemos un resumen de internet, fotocopiar un texto, escanear y en otros casos copia y pega. ¿Dónde queda el ejercicio de nuestras funciones cerebrales? ¿Cómo podemos trascender nuestros pensamientos si ya no hay un legado?

9. La administración del tiempo: Todo venezolano tiene derecho a la diversión y al esparcimiento, tenemos más de treinta (30) días no laborables al año, más de cien (100) fines de semana, sábados y domingos que por ley son los dos días no laborables, un mes de vacaciones por año, reposos médicos, viajes, problemas de transporte, aniversarios y un sinfín de eventos que suman cada vez más razones paro no asistir a clases o estudiar. El estudiante venezolano estudia el día antes del examen o de la exposición, elabora el trabajo el día antes de la presentación. Son muy pocos 
los casos del docente que enseña las técnicas de estudio y la administración del tiempo del estudiante. El estudiante venezolano "Va a la escuela, va al liceo o va a la universidad", quien realmente va a estudiar, quien realmente se prepara, es un porcentaje muy bajo en la población estudiantil y su labor ejemplar es considerada nerd o cerebrito, que al igual que sus compañeros o a la mayoría de los estudiantes debe, aunado a ello, trabajar en proporcionarse los medios de sustento humano: comida, vivienda y vestido, aunado a las nuevas necesidades: transporte y telefonía sin descartar que se debe garantizar su seguridad. La prioridad es cualquier cosa y en cuanto al estudio se oye la frase: "profesor(a) le puedo hacer un trabajo rapidito..." y en parte del proceso educativo queda la enseñanza, el aprendizaje, la didáctica, la trasmisión del conocimiento. La teoría del Constructivismo y el Cognitivismo tiene grandes aportes en la educación, pero la transmisión de valores y la impresión de virtudes no se puede plasmar en un "trabajo rapidito".

10. La mala cultura del uso del internet: En este punto final de los enumerados, como los principales problemas que no permiten aumentar la calidad educativa en Venezuela, se considera a este como el núcleo de los nueve puntos anteriores. El mal uso del internet ha transculturizado al estudiante, hace que el estudiante y el joven venezolano pase horas $\mathrm{y}$ horas conectado a las redes sociales que repiten y transmiten informaciones que carecen de fundamento de sustento. Colocar el estado de ánimo permite a los otros apreciar cómo, supuestamente, es el estado de quien está en una red social determinada con el fin de que si no estás en internet, NO EXISTES. Tu banco, tus servicios, tus amigos todo está conectado al internet, pero los conecta tanto que se
DESCONECTAN de la realidad. Privar a un estudiante, niño o joven venezolano de acceder a sus redes sociales o privarlo del uso de su teléfono celular es un CASTIGO sin precedentes para la juventud del 2017. El joven venezolano, puede pasar el día entero en cualquier espacio físico sin interactuar, con nada más que su teléfono o el internet. Observe cualquier evento social y vera como un alto porcentaje de las personas están concentradas en su teléfono. ¿Qué amerita tanto tiempo de las personas para estar sumergidos en internet? 1. Ver los estados de otras personas en cada red social. 2 . Reproducción de videos de todo tipo y género de acuerdo a la tendencia del usuario. Y el 3 y más peligroso REDIFUNDIR los mensajes que a criterio del usuario son llamativos sin importar la fuente, el contenido intrínseco del mensaje, ni siquiera confirmar la veracidad de dichos contenidos.

Es el internet un gran avance para la tecnología y para la comunicación en red, pero no hemos sido educados para emplear esta herramienta de manera adecuada, no hay una administración eficiente del tiempo mientras se navega y se comparte en las redes sociales, el tiempo pasa rápidamente y obviamos lo fundamental de la educación y de la vida social, compartir con nuestros semejantes.

\section{Propuestas que contrarrestan la gerencia educativa en la actualidad}

Enumerados los 10 puntos reales que afectan la educación en Venezuela para el año 2017, a continuación, se proponen ocho aspectos a considerar, teniendo la premisa de los aspectos más resaltantes de la educación de los países que son modelo de excelencia educativa. Considerando que los venezolanos tenemos las capacidades y condiciones que pocos países tienen: diversidad de recursos no renovables, diversidad de ecosistemas, población joven predominante y un legado de 
historia que nos ha hecho comprender como dijo Simón Bolívar: que nos han dominado más por la ignorancia que por la fuerza.

Los siguientes puntos o consideraciones son de carácter individual, no son para el gobierno de turno ni para una institución específica, son para que el lector, si es venezolano o vive en este país, dé su aporte como ciudadano y germine la semilla de valores y actos que van desde el estoicismo hasta la soberanía patria.

1. Ame a su familia: haga de su núcleo familiar el centro de amor, afecto y respeto donde giren todos los aspectos de su vida. Para ello usted no está solo, existen numerosas instituciones que están dispuestas a orientarlo y ayudarle en este aspecto que, aunque se lee sencillo es muy complejo. Para amar existen tres premisas fundamentales que los estereotipos, artistas y medios de comunicación hacen ver como imposibles, ellos son:

- Aprenda a perdonar.

- Integre la religión a su vida familiar.

- Acepte a las personas como son.

Se leen extraordinariamente fácil, ahora haga una introspección y podrá descubrir que hay cosas que usted no perdona, vera que usted ha sentido rabia con los miembros de su familia e incluso hemos ofendido y herido moral y físicamente a nuestros seres queridos. Qué difícil es ser fiel a la pareja, que difícil es obedecer a nuestros padres, que difícil es escuchar a nuestros hijos y está escrito en las sagradas escrituras $y$ en cualquier libro de superación personal: Trate de llevar una vida ejemplar y todo, absolutamente todo se le resume en los diez mandamientos de la Biblia. ¿Usted no es católico ni cristiano? Lea la Tora, el Corán, pero permita que el espíritu divino guíe sus pasos.

2. Desarrollo personal: Nunca deje de estudiar, no pase un día sin leer, retome las lecturas de las obras clásicas: La Ilíada, Don
Quijote de la Mancha, Doña Bárbara, La Biblia. Lea diariamente 5 palabras del diccionario. Investigue, desarrolle, asista a conferencias, foros, talleres de los temas de su interés, pero no abandone las aulas por mucho tiempo. Apruebe su bachillerato, apruebe su pre grado, estudie una maestría, un doctorado. No pierda la valiosa herramienta del aprender.

3. Trate a la gente con respeto: Los niños son como esponjas para aprender y adquirir modelos de conducta, si tus hijos observan tu forma respetuosa de dirigirte a la gente ellos siempre tratarán de imitarte. Aun cuando una persona se encuentre en estado de exaltación, ser respetuoso y cortés siempre abre las puertas y hace que la gente te trate con una percepción diferente.

4. Elabore un cronograma de sus actividades: Visualizar las actividades diarias permite llevar un mejor control de las actividades que realiza a diario y podrá tener más tiempo y espacio para ejecutar aquellas actividades que siempre debe posponer por falta de tiempo.

5. Repase lo obvio: ¿Usted recuerda cuales son los elementos de una suma? ¿De una resta? ¿Recuerda usted la diferencia entre diptongo y hiato? ¿Desde cuándo no calcula una operación matemática con papel y lápiz? Estas cosas, aunque son básicas se nos olvidan ya que las cosas por sabidas se callan y por calladas se olvidan. Repase la historia, vuelva a leer los cuentos, libros y textos que leyó en el pasado. Con ello siempre reforzará su conocimiento.

6. Sea original: No aparente ser lo que no es, sea humilde. Cuando asistimos a lugares sociales vemos como los protagonistas del evento son los que tienen la ropa que está a la moda, el vehículo más costoso y que generalmente está acompañado de una hermosa dama (aparentemente, porque de seguro que su cuerpo ha sido sometido a 
intervenciones quirúrgicas y más seguro es que su tiempo de conocerse jamás excede de unos cuantos meses). Amigo lector, el protagonista de todo evento ES USTED y las personas que comparten con usted. Sea usted el escritor de su propia vida y haga que sea original, que no se parezca a nadie o si tiene un modelo a seguir que sea más por lo intelectual que por su apariencia y sus bienes.

7. Copie los mejores modelos y adáptelos a su realidad y al entorno: La innovación nos ayuda a mejorar todo proceso, sin embargo, no hace falta buscar la fórmula del agua tibia, busque en su entorno cuál es la familia más unida y más atenta y siga sus preceptos, busque el mejor maestro o profesor de su escuela y siga sus instrucciones, hágase su amigo y aplique los mismos procedimientos colocándole siempre su marca personal. Le exhorto a repasar las teorías pedagógicas: conductismo, constructivismo, cognitivismo, conectismo y todas aquellas que han demostrado cuán eficiente puede ser enseñar de acuerdo a los patrones de conducta y a las capacidades psicomotoras de su alumno. Estudie los fundamentos de otros países o regiones, evalúe cual es el mejor centro educativo de su estado, retómelos y adáptelos.

8. Planifique ejecute y actúe: Propóngase metas, la legislación venezolana a través de las comunas o consejos comunales y en compañía de los docentes, centros educativos y zonas educativas, pueden implementar medidas que satisfagan las necesidades de cada centro educativo. Lo vamos a repetir nuevamente, nuestras leyes y programas son perfectos, son holísticos y cumplen con toda la normativa lo necesario es que la comunidad, la familia y las instituciones en los niveles más bajas ACTÚEN y formen integralmente a los niños.
Los países asiáticos integran a sus estudiantes en las actividades de limpieza y trabajos de mantenimiento de las instituciones. Los docentes deben planificar este tipo de actividades y especializarse en cada una de sus áreas para plantear con fundamento cuando un alumno o estudiante alcanzó y/o aprobó las competencias necesarias para ser promovido.

La gerencia educativa en la actualidad no depende de un gobierno de turno, no depende de un ministro de turno, depende del trabajo integrado de los docentes, los padres o representantes y el compromiso de sus estudiantes. Para esto, es necesaria la dirección de un líder que promueva y contraste estos elementos que son en realidad la base de la educación. Sin embargo, la experiencia histórica demuestra que es en medio de las dificultades y las crisis, que los pueblos y las comunidades generan respuestas a sus vicisitudes. Nuestro país, Venezuela está en un período que requiere de acciones en los niveles más bajos. No sea usted el juez o el crítico. Sea usted un eslabón o la herramienta en este proceso de cambios tan necesarios para el desarrollo integral de la nación.

REFERENCIAS

Arteola (2010), Breve historia de la pedagogía. Disponible en http://brevehistoriadelapedagogia.blogspo t.com/

Beech, Jason. Y Brailovsky, Daniel. (2014) http://www.ibe.unesco.org/fileadmin/user _upload/COPs/Pages_documents/Compara tive_Research/AnalisisCompardo_ChinaCo noSur.pdf

Bempechat, J. (1990). The role of parental involmenten in children s academic achievement. New York.

Buenrostro, S., (2003) Educación en Japón, en Cinética, 23, agosto 2003, enero 2004. Disponible en: https://www.google.es/webhp?sourceid=c hromeinstant\&ion=1\&espv=2\&ie=UTF8\#q =Educaci $\%$ C3\%B3n $\% 20$ en $\% 20 J a p \% C 3 \% B$ 3n\%20Susuna\%20Buenrostro 
Chacón F., M. A. (2009). Metodología y Evaluación en la educación en el sistema educativo japonés, su visión holística $e$ integral. Disponible en: http://www.cidse.itcr.ac.cr/ciemac/memo rias/6toCIEMAC/Ponencias/Metodologia_y _Evaluacion_Japon_M_Chacon.pdfConsultad o:

Chikako F., (1993). El sistema educativo actual del Japón. En Revista de Relaciones Internacionales, $U N L P, \quad \mathrm{n} 4$, primer semestre, Disponible en: http://www.iri.edu.ar/revistas/revista_dv d/revistas/revista\%204/R4EST06.htmlCo nsultado:

Documental sobre la educación en Japón / documentary about japanese education

Fernández Montt René \& Luis Juan B. Clara (2010) Educación: La Influencia de los sistemas de creencias nómadas. Revista Crítica de Ciencias Sociales y Jurídicas 27 (3) pp.3-5. Recuperado de:https://pendientedemigracion.ucm.es/i nfo/nomadas/27/fernandezmontt_clara.pd $\mathrm{f}$

Froebel, Friedrich (s/f). La educación del hombre. Recuperado en http://www.biblioteca.org.ar/libros/8873 6.pdf

http://redormiga.org/educacion-en-espana

http://revistacultural.ecosdeasia.com/laeducacion-en-japon-una-aproximacion-alsistema-educativo-nipon/

http://www.aulaplaneta.com

http://www.china-family-adventure.com/eseducacion-en-china.html

http://www.dw.com/es/el-sistemaeducativo-alem\%C3\%A1n/a-2239631

http://www.justlanded.com/espanol/Alemani a/Guia-Alemania/Educacion/El-sistemaescolar-aleman http://www.mecd.gob.es/educacionmecd/areaseducacion/estudiantes/portad a.html;jsessionid=8DA86DF6B02FDB427F 2A117BF0A4703

http://www.universia.es/estudiarextranjero/espana/sistemaeducativo/estructura-sistemaeducativo/2892

https://www.youtube.com/watch?v=DkKmn5 TIDCI3 ene. 2013 - Subido por Colevisión Tv

La Educación en Japón. Una aproximación al Sistema Educativo nipón.

Mars, Amanda (2015). ¿Cuáles son las mayores economías del mundo? ¿Y las más diminutas?, El País (España). Disponible vía web

en: economia.elpais.com/economia/2015/04/ 15/actualidad/1429060990_180502.html

OCDE (2012). Resultados de PISA 2012 en Foco. Lo que los alumnos saben a los 15 años de edad y lo que pueden hacer con lo que saben. p. 5. Disponible en: www.oecd.org/pisa/keyfindings/PISA201 2_Overview_ESP-FINAL.pdf.

Pantoja, A. \& M. Alcaide. (2013). La variable género y su relación con el autoconcepto y el rendimiento académico del alumnado universitario. Revista Científica Electrónica De Educación y Comunicación en la Sociedad del Conocimiento. pisa-lo-quehicieron-

Redacción. Revista Semana (2014). El camino alemán, Revista Semana (Colombia). Disponible vía web en: www.semana.com/educacion/articulo/hac e-13-anos-alemania-se-rajo-en-laspruebas-pisa-lo-que-hicieron-paratransformar-esos-re. 